\title{
Correction to: Soybean iron deficiency chlorosis high throughput phenotyping using an unmanned aircraft system
}

\author{
Austin A. Dobbels and Aaron J. Lorenz
}

\section{Correction to: Plant Methods (2019) 15:97}

https://doi.org/10.1186/s13007-019-0478-9

In the original article [1], under the subheading "Image data processing", last paragraph, last sentence that reads as "The least data collection" was incorrectly published. The correct sentence should read as "Least-significant differences $(P<0.20)$ were calculated for all 36 trials on both ground-based and UAS-image based scores for both dates of data collection." The original article has been corrected.

The original article can be found online at https://doi.org/10.1186/s1300 7-019-0478-9.

Published online: 10 October 2019
Reference

1. Dobbels AA, Lorenz AJ. Soybean iron deficiency chlorosis high throughput phenotyping using an unmanned aircraft system. Plant Methods. 2019;15:97.

\section{Publisher's Note}

Springer Nature remains neutral with regard to jurisdictional claims in published maps and institutional affiliations. 